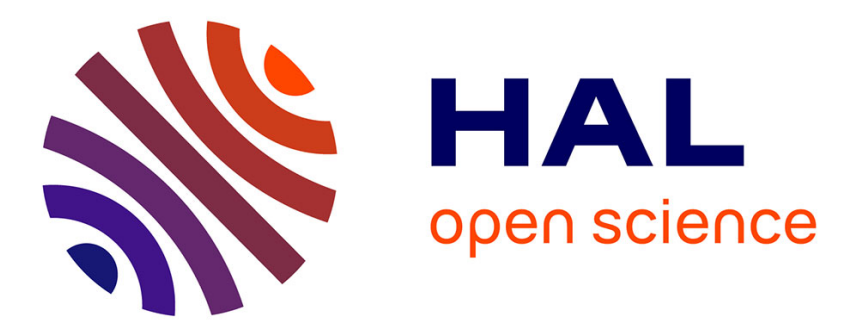

\title{
Multiple chiral topological states in liquid crystals from unstructured light beams
}

Charles Loussert, Etienne Brasselet

\section{To cite this version:}

Charles Loussert, Etienne Brasselet. Multiple chiral topological states in liquid crystals from unstructured light beams. Applied Physics Letters, 2014, 104 (5), pp.1911 (1-4). 10.1063/1.4864096 . hal-00950526

\section{HAL Id: hal-00950526 \\ https://hal.science/hal-00950526}

Submitted on 23 Feb 2016

HAL is a multi-disciplinary open access archive for the deposit and dissemination of scientific research documents, whether they are published or not. The documents may come from teaching and research institutions in France or abroad, or from public or private research centers.
L'archive ouverte pluridisciplinaire HAL, est destinée au dépôt et à la diffusion de documents scientifiques de niveau recherche, publiés ou non, émanant des établissements d'enseignement et de recherche français ou étrangers, des laboratoires publics ou privés.

\section{다(1) $\$$}

Distributed under a Creative Commons Attribution - NonCommercial| 4.0 International 


\title{
Multiple chiral topological states in liquid crystals from unstructured light beams
}

\author{
Charles Loussert and Etienne Brasselet ${ }^{\mathrm{a})}$ \\ Laboratoire Ondes et Matière d'Aquitaine, Univ. Bordeaux, CNRS, UMR 5798, F 33400 Talence, France
}

\begin{abstract}
It is shown experimentally that unstructured light beams can generate a wealth of distinct metastable defect structures in thin films of chiral liquid crystals. Various kinds of individual chiral topological states are obtained as well as dimers and trimers, which correspond to the entanglement of several topological unit cells. Self-assembled nested assemblies of several metastable particle-like topological states can also be formed. Finally, we propose and experimentally demonstrate an opto-electrical approach to generate tailor-made architectures.
\end{abstract}

Shaping materials and their properties are nowadays achieved from macroscopic down to nanometer-sized scales. A challenging issue remains the realization of controlled, hopefully reconfigurable, topological patterning of matter. In particular, when inversion symmetry is broken, nontrivial states can be protected in the sense that they cannot be transformed one to another by mere continuous changes of the material texture, which is relevant for information storage. Recent examples in condensed matter physics include the writing and deleting of (i) particle-like molecular ordering configurations in chiral nematic liquid crystals (i.e., cholesterics) films ${ }^{1}$ and (ii) particle-like spin configurations in magnetic films. ${ }^{2}$ In the latter case, the effect is triggered by local spin-polarized currents whereas optical vortex beams, which are themselves associated with topological features, are at play in the former case. Interestingly, in contrast to Ref. 2 that deals with a single topological state, three distinct nontrivial configurations have been reported in Ref. 1. However, their formation is achieved at the expense of an important prerequisite: the use of structured light beams carrying high-order optical phase singularities. Importantly, the absence of a bijection relationship between the topological charge of the pump vortex beam and the topological features of the generated structure suggests that the light-matter topological interplay is not a necessary condition for the appearance of chiral topological states in cholesteric liquid crystals.

Here, we unveil the generation of a variety of distinct metastable topological states in chiral nematic liquid crystals from unstructured light beams. The experimental demonstration is made by illuminating a thin chiral nematic film with a moderately focused (hence, paraxial) linearly polarized Gaussian laser beam. In addition, the optically written structures can be either reconfigured or erased by a low-voltage electric field. Various kinds of individual structures are obtained as well as multimeric topological states, which correspond to the entanglement of several topological building blocks. Moreover, we report on self-assembled nested superstructures. We also propose and demonstrate an optoelectrical approach to generate on-demand architectures.

The experiments are carried out using the setup shown in Fig. 1(a). A linearly polarized fundamental Gaussian laser

\footnotetext{
a)Email: e.brasselet@loma.u bordeaux1.fr
}

beam operating at $532 \mathrm{~nm}$ wavelength is moderately focused at normal incidence on the liquid crystal sample by a microscope objective $(20 \times$, numerical aperture 0.4$)$ used under overfilling conditions, which gives a beam diameter $2 w_{0}=1.6 \mu \mathrm{m}$ at $\exp (-2)$ of its maximum intensity. The sample consists in a $10 \mu \mathrm{m}$-thick cholesteric liquid crystal film sandwiched between two glass plates provided with transparent electrodes and prepared to promote perpendicular alignment of the director $\mathbf{n}$ a unit vector that defines the local average orientation of the molecules that constitute the liquid crystal. The material is prepared by doping the (achiral) nematic liquid crystal MLC-2048 by the chiral compound S811 (both from Merck) at 0.86 wt. \% concentration. This leads to a helical order with pitch $p=10.4 \mu \mathrm{m}$, which is the distance over which $\mathbf{n}$ rotates by $2 \pi$, as illustrated in Fig. 1(b). In the present case, the director at rest is unwound since $p$ is larger than the critical pitch $p_{c}=2 K_{2} d / K_{3}{ }^{3}$, where $K_{2}$ and $K_{3}$ are, respectively, the twist and bend Frank elastic constants of the liquid crystal, which gives $p_{c}=0.84 \mathrm{~d}^{4}$ Such a situation is an example of geometrical frustration of the supramolecular chiral ordering of cholesterics. ${ }^{5}$ The overall orientational ground state of the sample therefore corresponds to uniformly aligned director field along the normal to the plane of the film. Here, a Gaussian laser beam is used to locally relieve the frustration in a controlled fashion, which leads to the formation of particle-like chiral topological states.

In a first set of experiments, we select the lowest total incident power $P$ that generates a metastable defect structure when the sample is placed in the focal plane of the microscope objective (i.e., $z=0$ ). In practice, this corresponds to $P \sim 70 \mathrm{~mW}$ and the obtained structure, labeled $S_{1}$, is shown in Fig. 2(a), when observed between crossed polarizers after the laser beam is switched-off. This agrees with previous observations performed under moderate focusing for the writing light beam. ${ }^{6,7}$ Then, by placing the sample out of the focal plane, two other distinct metastable particle-like states $S_{2}$ and $S_{3}$ are identified for large enough values of $z$ and $P$, as shown in Figs. 2(b) and 2(c). The typical minimal values of $z$ and $P$ above which the structures $S_{2}$ and $S_{3}$ have been observed are $z \sim 30 \mu \mathrm{m}$ and $z \sim 80 \mu \mathrm{m}$, respectively, and correspond to $P \sim 300 \mathrm{~mW}$ and $P \sim 600 \mathrm{~mW}$. The reproducibility of these results are demonstrated in the bottom panels of Fig. 2 that display average images taken over more than ten 
(a) Focusing

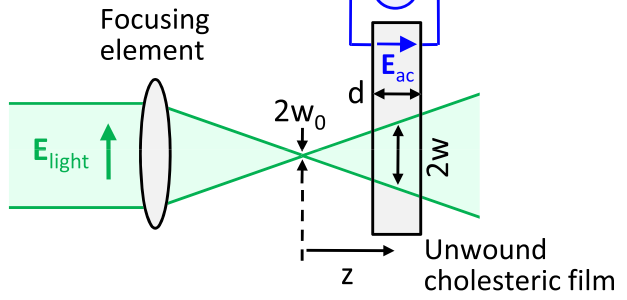

(b)

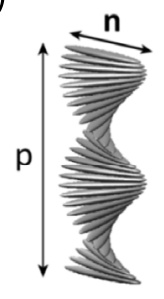

FIG. 1. (a) Experimental setup: A linearly polarized Gaussian light field $E_{\text {light }}$ impinges at normal incidence onto a unwound cholesteric film of thick ness $d$. The liquid crystal film is placed at a distance $z$ from the focal plane of the focusing element where the beam waist radius is $w_{0}$ whereas it is $w$ in the plane of the sample. An additional quasistatic electric field $E_{\text {ac }} \quad V / d$ can be applied along the normal to the sample. (b) Helical order of a chiral ne matic liquid crystal, where $p$ is the pitch of the helix and $\mathbf{n}$ is the director with double head arrow meaning invariance under $\mathbf{n} \rightarrow \mathbf{n}$.

independent events obtained under identical experimental conditions. Indeed, the difference between single and average images is hardly visible.

The fact that the structures $S_{1}, S_{2}$, and $S_{3}$ shown in Fig. 2 correspond to distinct, metastable, reconfigurable, and erasable topologically protected chiral states is experimentally inferred from Fig. 3. This figure presents the evolution of the diameter of the structures ${ }^{8}$ as a quasistatic applied voltage $V$ at frequency $f_{1}$ is increased by $0.05 \mathrm{~V}_{\text {rms }}$ incremental steps, noting that the nematic host has a positive dielectric anisotropy at this frequency. Namely, $\epsilon_{a}\left(f_{1}\right)=\epsilon_{\|}\left(f_{1}\right)-\epsilon_{\perp}\left(f_{1}\right)$ $=+3.2,{ }^{4}$ where $\epsilon_{\|, \perp}$ is the relative dielectric permittivity along directions parallel and perpendicular to $\mathbf{n}$, respectively. The director thus tends to align along the applied electric field, thereby progressively unwinding the three-dimensional distorted orientational state. Importantly, the electrical unwinding exhibits a step-like behavior that emphasizes both the energy barriers between the structures and their hierarchical nature. Indeed, successive sharp transitions between the structures are observed before all of them are eventually unwound at large enough applied voltage (Fig. 3). Namely, we identify the sequences $S_{3} \rightarrow S_{2} \rightarrow S_{1} \rightarrow S_{0}, S_{2} \rightarrow S_{1} \rightarrow$ $S_{0}$ and $S_{1} \rightarrow S_{0}$, where $S_{0}$ refers to the unwound orientational ground state.
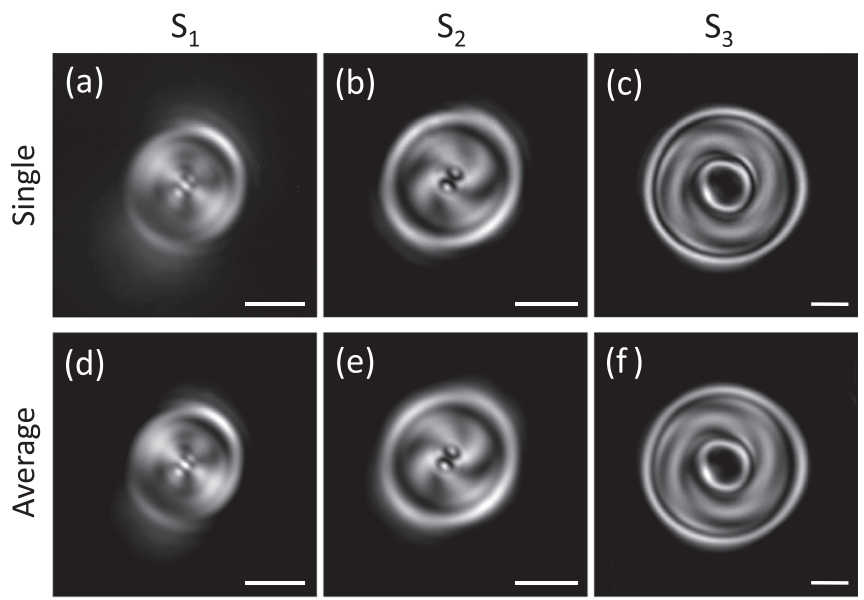

FIG. 2. White light crossed linear polarizers imaging of three distinct kinds of chiral topological states labeled $S_{1}$ [panels (a and d)], $S_{2}$ [panels (b and e)], and $S_{3}$ [panels (c and f)], see text for details. Upper row refers to individ ual states whereas bottom row presents average images taken over more than ten independent defect structures. Scale bar is $10 \mu \mathrm{m}$.

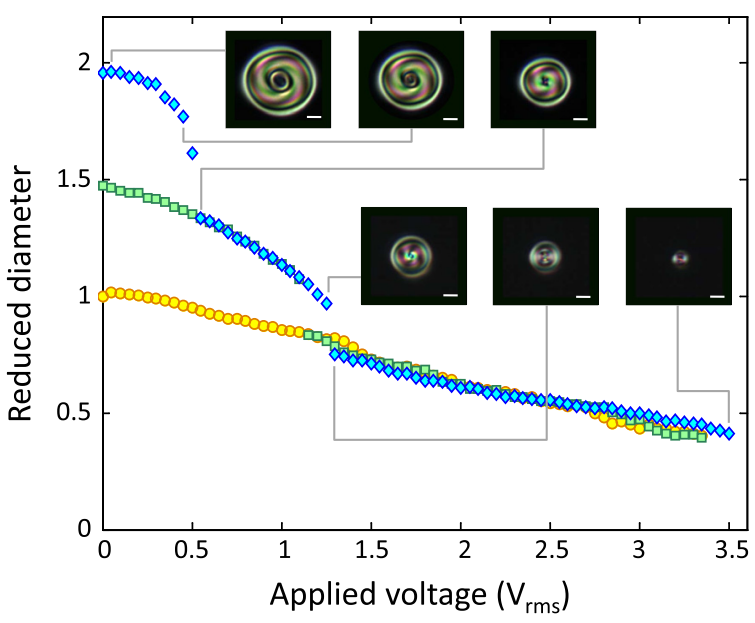

FIG. 3. Diameter of the three structures presented in Fig. 2 as a function of the applied voltage $V$ at frequency $f_{1} \quad 1 \mathrm{kHz}$, for which $\epsilon_{a}>0 . S_{1}$ : circle markers; $S_{2}$ : square markers; and $S_{3}$ : diamond markers. The diameters are normalized to the diameter of $S_{1}$ at $V \quad 0$. Scale bar is $5 \mu \mathrm{m}$.

Noticeably, both the main features of the structures $S_{n}$ ( $n=1,2$, and 3 ) described above and their visual inspection strikingly match with the triple-twisted particle-like elastic excitations generated by Laguerre-Gaussian beams previously reported by Smalyukh et al. ${ }^{1}$ This invites to identify the structures $S_{n}$ as the T3- $n$ "torons" introduced in Ref. 1 even though the three-dimensional reconstruction of the director field has not carried out in this work. Also, we stress the lack of available data in the literature regarding the structures T3-2 and T3-3 that are only reported in Ref. 1, which deals with highly nonparaxial Laguerre-Gauss writing light fields, despite the large number of studies that followed. ${ }^{1,6,7,9-13}$ Hopefully, this work will contribute to fill this gap.

The important conclusion drawn from above results is that the use of structured light, such as Laguerre-Gaussian vortex beams, is not a necessary condition to the formation of various chiral topological states as previously established in Ref. 1, where T3-1 was found to be associated to Laguerre-Gauss beams $\mathrm{LG}_{0 \ell}$ with $0 \leq|\ell| \leq 4$, T3-2 to $4 \leq|\ell| \leq 8$, and T3-3 to $|\ell|>8$. Still, a unified picture to explain present (Gaussian excitation) and previous (Laguerre-Gaussian excitation) experimental observations would be desirable. This is done by recalling that (i) the typical size of the spatial extent of a doughnut-shaped $\mathrm{LG}_{0 \ell}$ beam $(\ell>0)$, namely, the diameter of the circle of its maximum intensity $D_{\ell}=w_{0} \sqrt{ } 2 \ell$, increases with $\ell$ and (ii) the structures $S_{1}, S_{2}$, and $S_{3}$ successively appear for increasing values of Gaussian beam diameter in the plane of the sample. It is therefore the characteristic spatial extent of the writing beam that is likely to be at work in the laser-induced formation of the particle-like chiral topological states rather than the intrinsic structural features of the excitation light field.

In a second set of experiments, we explored the case of much larger excitation beam diameter, typically, ten times larger than those used in Figs. 2 and 3, which requires to increase the total incident optical power up to a few Watts. By doing so, various kinds of exotic structures may appear such as those shown in Fig. 4 where single [panels (a, d, and g)], dimer [panels (b, e, and h)], and trimer [panels (c, f, and i)] defect structures are shown. More precisely, the leftmost 

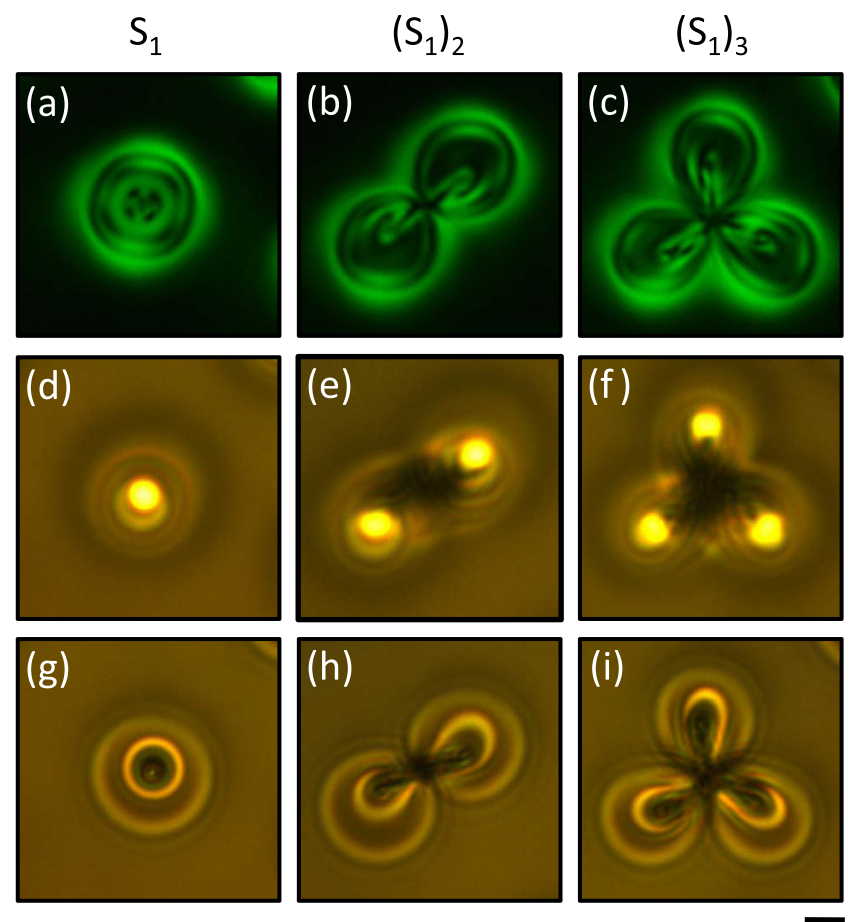

FIG. 4. Metastable single [ $S_{1}$, panels (a, d, and g) ], dimer $\left[\left(S_{1}\right)_{2}\right.$, panels (b, e, and $\mathrm{h})]$, and trimer $\left[\left(S_{1}\right)_{3}\right.$, panels (c, f, and i) $]$ structures, see text for details. Upper row: crossed linear polarizers imaging at $532 \mathrm{~nm}$ wavelength. Middle and bottom rows: natural light imaging in two different planes. Scale bar is $5 \mu \mathrm{m}$.

column of Fig. 4 corresponds to the $S_{1}$ state whereas the two other columns correspond to multimeric topological states made of several building blocks of the $S_{1}$ type, namely, the dimer $\left(S_{1}\right)_{2}$ and the trimer $\left(S_{1}\right)_{3}$. By looking at these structures in two different imaging planes, it is possible to illustrate some of their intriguing characteristics. For instance, Figs. 4(d) 4(f) emphasize the focusing properties of each building blocks, hence the multi-lens nature of a multimer. On the other hand, Figs. 4(g) 4(i) allow to identify the entanglement between individual topological structures that self-assemble into multimers, as illustrated by the $n$-arm "defect skeleton" of $\left(S_{1}\right)_{n}$. Interestingly, we note that self-assembled chiral dimer structures have also been reported a few years ago by Tkalec et al. ${ }^{14}$ in a colloidal suspension hosted in a so-called twisted nematic film, which is achiral at the molecular scale and chiral at the supramolecular scale. However, both situations drastically differ since the latter case corresponds to solid colloidal particles bounded by an external liquid crystal defect, whereas our case represents an all-liquid version where particle-like structures share a complex defect network whose topological nature remains to be identified.
Another kind of self-assembled structures is also observed under experimental conditions similar to those leading to multimers, namely, discrete sets of $S_{1}$ structures embedded inside a larger loop defect. This is illustrated in Fig. 5 for $N=1$ [Fig. 5(a)], $N=2$ [Fig. 5(b)], $N=3$ [Fig. 5(c)], $N=7$ [Fig. 5(d)], and $N=17$ [Fig. 5(e)] close-packed $S_{1}$ structures. Intriguingly, such metastable architectures mimic optimal solutions to the problem of dense packing of congruent circles in a circle, ${ }^{15}$ at least up to $N=7$ from Fig. 5. This suggests that $S_{1}$ structures formally behave as nondeformable objects with circular crosssections; hence, emphasizing both their particle-like and topological protection features. From an application point of view, this gives access to various kinds of symmetries without need of creating them "point-by-point" as reported so far. ${ }^{10}$ In particular, hexagonal periodic symmetry is found for $N=7$ [Fig. 5(d)], whereas aperiodic two-dimensional distributions can be seen for $N=17$ [Fig. 5(e)]. However, such nested topological assemblies basically result from a complex spontaneous process that prevents from getting a prescribed value of $N$ in a controlled fashion. With the aim at coping with this issue, hereafter, we propose and demonstrate experimentally an opto-electrical option.

The main idea is to benefit from (i) the on-demand alloptical generation of $S_{n}$ structures, as presented in Fig. 2 and (ii) the electrical reconfiguration capabilities of a liquid crystal, which are especially attractive for so-called dual frequency nematic materials that exhibit either positive or negative dielectric anisotropy depending on the applied voltage frequency. In fact, this is the case for the nematic host used here, whose dielectric anisotropy is negative at frequency $f_{2}=100 \mathrm{kHz}$, namely, $\epsilon_{a}\left(f_{2}\right)=-3.4$ (Ref. 4) whereas $\epsilon_{a}>0$ can be achieved below a critical frequency, as already exploited for Fig. 3. Practically, a Gaussian laser beam alone is first used to generate a structure $S_{3}$ [Fig. 6(a)]. Then a quasistatic electric field at frequency $f_{2}$ is applied, which leads to a controlled stretching of the structure into a loop defect whose diameter increases with the applied voltage, as shown in Fig. 6(b) where $V=0.45 \mathrm{~V}_{\text {rms }}$. The next step consists to the optical formation of a structure $S_{1}$ inside the loop, where the director field is unwound, while maintaining the electric field [Fig. 6(c)]. The system eventually relaxes without any external fields, which eventually leads to self-centered, metastable, nested topological states [Fig. 6(d)]. Obviously, the proposed approach could be straightforwardly generalized to arbitrary tailor-made self-close-packed architectures.

By focusing on localized defect structures obtained under non-topological electromagnetic fields, present work
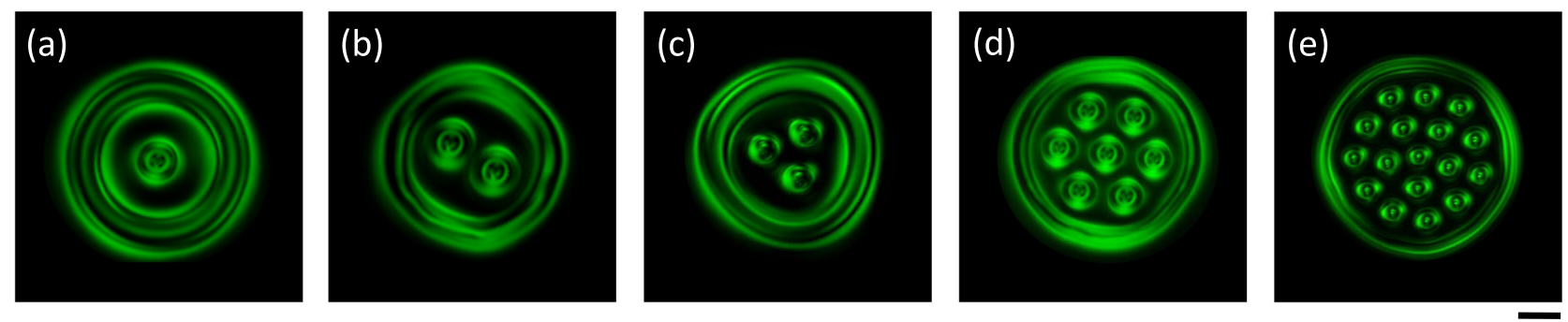

FIG. 5. Metastable self assembled multiple $S_{1}$ structures embedded inside a larger loop defect, see text for details. Here, are shown the cases of $N \quad 1$ (a), $N \quad 2$ (b), $N \quad 3(\mathrm{c}), N \quad 7$ (d), and $N \quad 17$ (e) close packed $S_{1}$ structures. Images correspond to crossed linear polarizers imaging at $532 \mathrm{~nm}$ wavelength. Scale bar is $15 \mu \mathrm{m}$. 

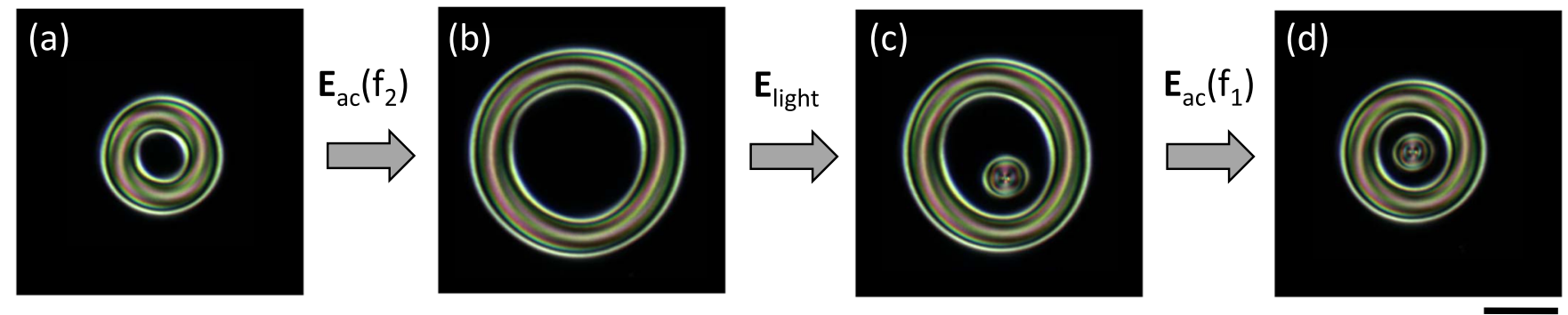

FIG. 6. Principle of the opto electrical generation of particle like topological states embedded inside a defect loop. First, a structure $S_{3}$ is optically generated [panel (a)], then electrically stretched by applying a quasistatic voltage at frequency $f_{2}$, for which $\epsilon_{a}<0$ [panel (b)]. A structure $S_{1}$ is then optically induced inside the loop while the applied voltage is maintained [panel (c)]. The system eventually relaxes into a metastable nested state without any external fields [panel (d)]. Images correspond to white light crossed linear polarizers imaging. Scale bar is $30 \mu \mathrm{m}$.

thus extends prior art of field-induced three-dimensional chiral structures. Indeed, it is known since four decades that defect domains can be generated in frustrated cholesteric films, though without spatial control, by applying uniform, hence unstructured, quasistatic electric fields see Ref. 16 for a review an early example being the case of the socalled "spherulites." 17,18

To conclude, it has been experimentally unveiled that unstructured light beams such as uniformly polarized Gaussian laser modes can generate a broad range of metastable topological states in chiral nematic liquid crystals. Such states, which consist in particle-like three-dimensional elastic excitations, have also been demonstrated to self-assemble in various ways. For instance, multimeric and nested topological superstructures of different symmetries have been reported. An opto-electrical strategy to generate tailor-made self-close-packed patterns has also been proposed and demonstrated experimentally. By disclosing novel kinds of topologically protected chiral states in a soft matter system that is inherently optically anisotropic and nonlinear, these results might lead to intriguing singular photonics phenomena and applications as well as developments regarding the study and use of topology in condensed matter physics.

This work was financially supported by DGA Contract No. 201160083.

${ }^{1}$ I. I. Smalyukh, Y. Lansac, N. A. Clark, and R. P. Trivedi, Nat. Mater. 9, 139 (2010).
${ }^{2}$ N. Romming, C. Hanneken, M. Menzel, J. E. Bickel, B. Wolter, K. von Bergmann, A. Kubetzka, and R. Wiesendanger, Science 341, 636 (2013).

${ }^{3}$ B. Y. Zel'dovich and N. V. Tabiryan, JETP Lett. 34, 406 (1982).

${ }^{4}$ J. S. Hsu, B. J. Liang, and S. H. Chen, Appl. Phys. Lett. 89, 051920 (2006).

${ }^{5}$ R. D. Kamien and J. V. Selinger, J. Phys.: Condens. Matter 13, R1 (2001).

${ }^{6}$ I. I. Smalyukh, D. Kaputa, A. V. Kachynski, A. N. Kuzmin, P. J. Ackerman, C. W. Twombly, T. Lee, R. P. Trivedi, and P. N. Prasad, Opt. Express 20, 6870 (2012).

${ }^{7}$ B. Yang and E. Brasselet, J. Opt. 15, 044021 (2013).

${ }^{8}$ The diameter of the structure is experimentally determined as the diameter of the largest bright ring of its image observed between crossed circular polarizers at $532 \mathrm{~nm}$ wavelength. Indeed, crossed circular polarizers imag ing of an axisymmetric birefringent and inhomogeneous pattern leads to an axisymmetric pattern whose characteristic size can be assessed in a reli able manner.

${ }^{9}$ O. Trushkevych, P. Ackerman, W. A. Crossland, and I. I. Smalyukh, Appl. Phys. Lett. 97, 201906 (2010).

${ }^{10}$ P. J. Ackerman, Z. Qi, and I. I. Smalyukh, Phys. Rev. E 86, 021703 (2012).

${ }^{11}$ P. J. Ackerman, Z. Qi, Y. Lin, C. W. Twombly, M. J. Laviada, Y. Lansac, and I. I. Smalyukh, Sci. Rep. 2, 414 (2012).

${ }^{12}$ J. S. Evans, P. J. Ackerman, D. J. Broer, J. van de Lagemaat, and I. I. Smalyukh, Phys. Rev. E 87, 032503 (2013).

${ }^{13}$ B. G. g. Chen, P. J. Ackerman, G. P. Alexander, R. D. Kamien, and I. I. Smalyukh, Phys. Rev. Lett. 110, 237801 (2013).

${ }^{14}$ U. Tkalec, M. Ravnik, S. Zumer, and I. Musevic, Phys. Rev. Lett. 103, 127801 (2009).

${ }^{15}$ R. Graham, B. Lubachevsky, K. Nurmela, and P. Osterggard, Discrete Math. 181, 139 (1998).

${ }^{16}$ P. Oswald, J. Baudry, and S. Pirkl, Phys. Rep. 337, 67 (2000).

${ }^{17}$ W. E. L. Haas and J. E. Adams, Appl. Phys. Lett. 25, 263 (1974).

${ }^{18}$ M. Kawachi, O. Kugure, and Y. Kato, Jpn. J. Appl. Phys., Part 1 13, 1457 (1974). 